\title{
The spoke brightness illusion originates at an early motion processing stage
}

\author{
ALEX O. HOLCOMBE \\ Harvard University, Cambridge, Massachusetts \\ JAMES INTRILIGATOR \\ Beth Israel Deaconess Medical Center, Boston, Massachusetts \\ and \\ PETER U. TSE \\ Max Planck Institute for Biological Cybernetics, Tübingen, Germany
}

\begin{abstract}
When a bright white disk revolves around a fixation point on a gray background, observers perceive a "spoke": a dark gray region that connects the disk with the fixation point. Our first experiment suggests that motion across the retina is both necessary and sufficient for spokes: The illusion occurs when a disk moves across the retina even though it is perceived to be stationary, but the illusion does not occur when the disk appears to move while remaining stationary on the retina. A second experiment shows that the strength of the illusion decreases with decreasing luminance contrast until subjective equiluminance, where little or no spoke is perceived. These results suggest that spokes originate at an early, predominantly luminance-based stage of motion processing, before the visual system discounts retinal motion caused by smooth pursuit.
\end{abstract}

When a bright white disk revolves around a fixation point on a gray background, observers report that a shadowy dark gray region appears to connect the disk with the fixation point (Holcombe, Macknik, Intriligator, Seiffert, \& Tse, 1999; Tse, 1997). A revolving black disk yields a similar illusory region that appears brighter than the background. We have coined the term spokes to refer to these illusory regions. When viewing such displays, observers also report illusory regions that extend beyond the disks. These are examples of the "wakes" illusion described in Holcombe et al. (1999).

Spokes appear when the disks begin moving, disappear when the disks stop moving, and do not appear when the disks flicker without moving. As a motion-induced brightness illusion, spokes may provide new clues to how motion and brightness are processed. We hope to eventually determine the role of the spoke-generating process in normal vision, its functional location in the visual processing stream, and its neural correlate.

In this paper we focus on the functional location of the spoke-generating process in the visual processing stream. In particular, we examine different types of motion to de-

We thank Patrick Cavanagh and Ken Nakayama for providing facilities and support for the studies, and Adriane Seiffert for comments on the manuscript. A.O.H. was supported by an NIMH NRSA graduate fellowship, and P.U.T. was supported by AASERT Grant F49620-940376. Correspondence should be addressed to A. O. Holcombe, Department of Psychology, Harvard University, 33 Kirkland St., Cambridge, MA 02138 (e-mail: holcombe@wjh.harvard.edu). termine which yield the illusion. In Experiment 1, we investigate whether motion of a disk across the retina, even if the disk is not perceived to move, is sufficient to cause the illusion. At late stages in motion processing, retinal motion due to smooth pursuit eye movements is discounted by the visual system. This discounting underlies our ability to perceive stationary objects veridically (although they across the retina) and our ability to perceive tracked objects as moving (even though they do not move across the retina) (see, e.g., Anstis \& Gregory, 1965; Erickson \& Thier, 1991). If the illusion is generated at a stage before this discounting process, a spoke should be perceived when motion across the retina occurs due to eye movements alone. However, if the illusion is generated at a stage after this discounting process, a spoke should not be perceived. In addition, if the illusion originates after the discounting process, then a disk moving in tandem with smooth pursuit eye movements should yield a spoke even though such a disk remains stationary on the retina.

\section{EXPERIMENT 1}

The displays used are schematized in Figure 1. The "reference" display consisted of a white disk moving in a circular path around a fixation point. The presence of a second figure besides the disk (in this case, the fixation point) is helpful to guide the eyes but is not necessary for the illusion. In an informal experiment only the disk was present, observers fixated empty space instead of a fixation point, and the illusion was still perceived. In the present experiment, observers were asked to judge the strength 


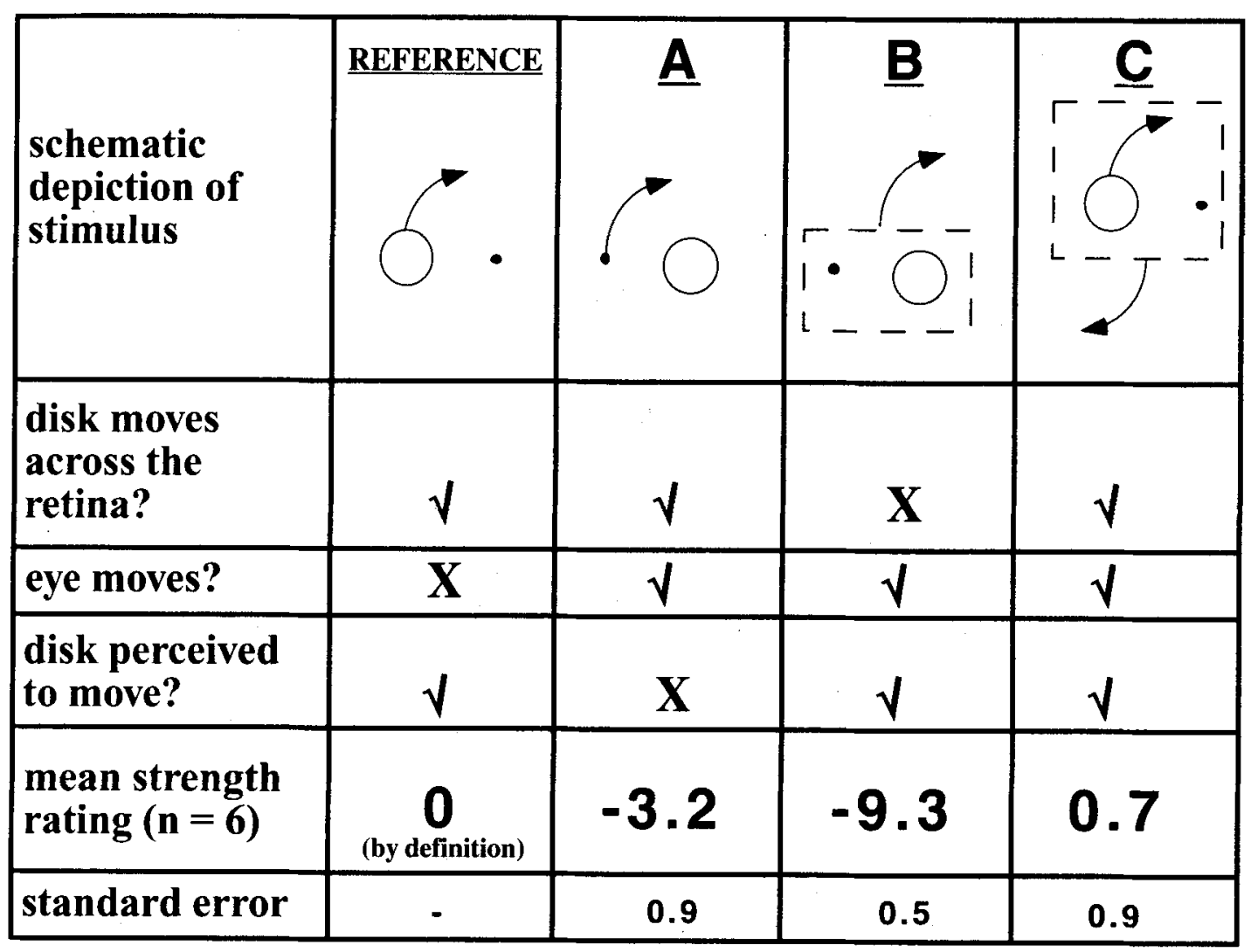

Figure 1. Schematic depiction of the stimulus displays and the results of Experiment 1. Observers fixated the small dot and rated the strength of the spoke caused by each of the displays relative to the strength of the spoke perceived in the reference display, on a scale of -10 to $10:-10$ if no spoke was perceived, 0 if the strength of the spoke was equal to the reference display, and 10 if it was as strong as could be imagined. In Display $B$, observers ${ }^{+}-9.3$ mean rating indicates that the spoke was very weak or absent, despite the fact that observers perceived the disk to move in the same path as in the reference display. In Display $A$, the disk moved across the retina, and even though observers did not perceive the disk to move, the spoke was perceived to be much stronger than in Display $B$. These results suggest that motion of a figure across the retina is necessary and sufficient for the illusion.

of spokes in the experimental displays relative to the strength of spokes in the reference display.

The experimental display denoted " $A$ " in Figure 1 consisted of a fixation point that moved around a central white disk. To the extent that observers accurately track the fixation point, the retinal stimulation caused by this display should be the same as that caused by the reference display. However, in these conditions the motion system discounts retinal motion due to eye movements, so the disk is perceived to move very little if at all. Thus, if the spoke illusion arises at a late stage in motion processing, spokes should be extremely weak or absent in Condition A. Alternatively, if the illusion arises at a stage that takes only retinal motion as input, then it should be strong in this condition.

In Display B, the fixation point and the large disk did not move relative to each other. Instead, they moved in tandem in a circular path. Their path had the same radius as the disk's path in the reference condition. When observers tracked the moving fixation point in this case, the disk did not move across the retina, but nevertheless it was perceived to move. If the spoke illusion arises at a late stage of motion processing, a spoke should be perceived in this condition.

Display C was a combination of Displays A and B: The disk and the fixation point moved together in a circular path, as in Display B, but in addition the disk revolved around the fixation point at the same time. Thus the disk moved both in the world and on the retina. To the extent that an observer's eyes pursued the fixation point accurately, the retinal motion of the disk was equivalent to that of the reference display, as well as to Display A. Therefore, if motion across the retina determines the strength of spokes, the strength of the illusion should be similar in Displays A and C.

\section{Method}

Participants. Six observers with normal or corrected-to-normal visual acuity participated. One had extensive experience observing these displays (A.H.), 4 others had extensive experience in psychophysical experiments and had seen the spoke illusion before, and 1 was entirely naïve (O.J.). 
Apparatus and Stimuli. Stimuli were displayed on a 13-in. Apple color monitor with a $66-\mathrm{Hz}$ refresh rate. Viewing distance was approximately $63 \mathrm{~cm}$, although head position was not physically constrained. All stimuli consisted of a filled white disk $\approx 2^{\circ}$ in diameter and $\approx 85.5 \mathrm{~cd} / \mathrm{m}^{2}$ in luminance, the center of which was always $\approx 3.4^{\circ}$ away from a smaller white fixation point, $\approx 4$ are min in diameter and $\approx 85.5 \mathrm{~cd} / \mathrm{m}^{2}$, with the rest of the screen approximately a uniform $1 \mathrm{~cd} / \mathrm{m}^{2}$. The spatial pattern of the displays was as described in the introduction and schematized in Figure 1. In each condition the disk, the fixation point, or both moved in a circular path around the screen or relative to each other at $\approx 5.54^{\circ} \mathrm{visual}$ angle $/ \mathrm{sec}$ (which was equal to $318^{\circ}$ of the circular orbit per second). The distance between the center of the disk and the center of the fixation point was always $\approx 1^{\circ}$. The direction of revolution was counterbalanced across observers. In Display C, the disk's revolution around the fixation point and the fixation point's revolution around its center were in phase, so that when the fixation point was at the highest point in its orbit, so was the disk. Since they traveled at the same speed, their rates of revolution were also equal. The experiment was conducted in a room that was fairly dark, but bright enough that the observers could see the frame of the computer screen and the other objects near them. This illumination was provided because without a stable reference frame, as in complete darkness, when a pursuit eye movement is made, the motion due to eye movements is not completely discounted and observers actually perceive small, stationary stimuli to move in the opposite direction (Bridgeman, 1995; Wertheim, 1987).

Procedure and Design. Observers first viewed the reference display and were asked whether they perceived a region darker than the background between the moving disk and the fixation point (i.e., a spoke). All observers reported that they did. To gain qualitative insight into the nature of the perceived spokes, each observer was then asked to draw the dark region he perceived on top of a static duplicate of the stimulus using the Canvas drawing software (www.deneba.com). Observers were not under time pressure and were allowed to switch freely between the drawing and the moving display. After completion of the drawing, the observers were shown each of the other displays for several seconds, in pseudorandom order. The observers were told that they were to judge the strength of the spoke that they perceived in each display relative to the strength of the spoke in the reference display. They were to respond with -10 if they perceived no spoke at all, 0 if they perceived a spoke equally as strong as the reference display's spoke, and 10 if the spoke was extremely high contrast and appeared to be drawn in. Next, the observers were allowed to freely view and switch among all four displays as they made their strength ratings.

\section{Results and Discussion}

Figure 2 shows each observer's drawing of the spoke perceived in the reference display. Some observers reported that the spokes did not have sharp edges, but nonetheless they agreed that their drawings were an accurate approximation of the spoke's shape. The drawings show that for each observer, the spoke extended from the inducing figure up to the fixation point but not beyond (see Holcombe et al., 1999, for more about this property).

Tabulated in Figure 1 are the mean strength ratings and corresponding standard errors for each of the conditions. For Display B, in which the disk did not move across the retina but did move in the world, the mean rating was -9.3 , indicating that the spoke illusion was absent or nearly so, even though the disk was perceived to move in a circular

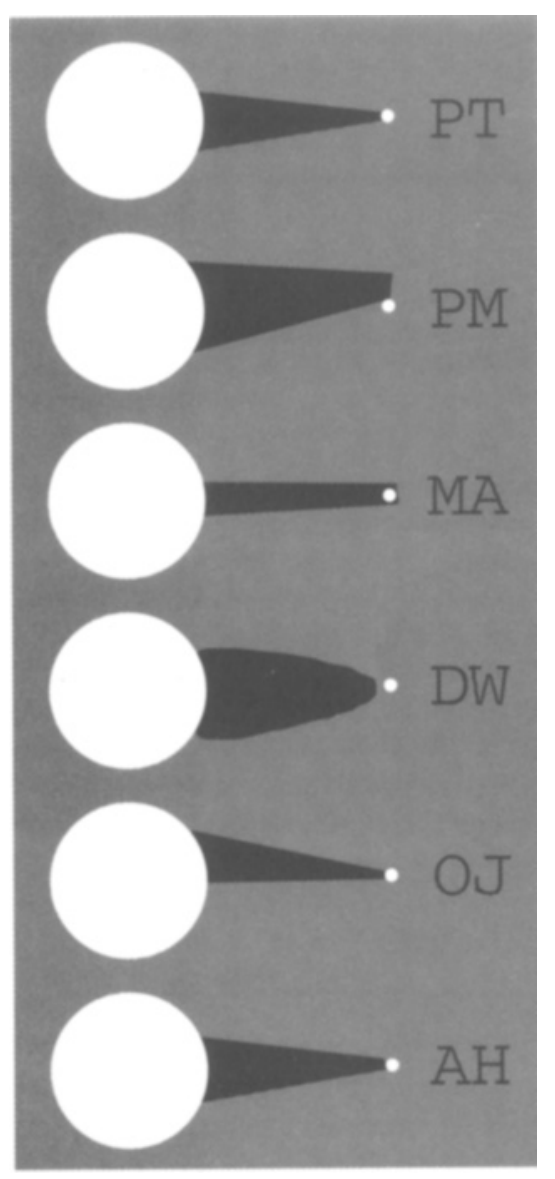

Figure 2. Observers' drawings of the spokes perceived in the reference display (depicted in figure) of Experiment 1. The white disk rotated clockwise around the fixation point. Over a static copy of the stimulus, observers used the polygon tool of the Canvas drawing software to draw the spoke perceived when the disk was directly to the left of the fixation point. Observers did not indicate the brightness of the spoke; the brightnesses in the figure are only schematic. Visit www.wjh.harvard.edu/ holcombe for a demonstration of the illusion.

path. The absence or near absence of spokes in this condition suggests that spokes are generated at a stage before pursuit-induced retinal motion is largely discounted to yield perceived motion. While viewing Display A, observers smoothly pursued in a circular path around a stationary disk and the spoke illusion did occur, with a mean rating of -3.2 , despite the fact that the disk was perceived to move little if at all in this condition. Together these two results imply that the spoke-generating process is driven by motion across the retina rather than by the motion perceived. However, the spokes of Display A were weaker than those of the reference display. This difference in strength may have been due to inaccuracy in observers' smooth pursuit performance, for such inaccuracy would have resulted in less smooth retinal motion in Display A. 
In particular, pauses in eye tracking would have resulted in intervals without retinal motion, and corrective saccades would have resulted in discontinuities in the motion.

Ratings of the spokes' strength in Display $\mathrm{C}$ were within $1 S E$ of the reference display's ratings. This provides further support for the hypothesis that the spoke illusion is driven by motion across the retina, as retinal motion should have been identical in Display $\mathrm{C}$ and the reference display. Note that eye movement inaccuracies would have been less likely to reduce the retinal motion in Display $C$ than in Display A, for even if eye movement stopped in Display $\mathrm{C}$, the continued motion of the disk in the world would have ensured continued retinal motion. This may be why the spokes of Display $C$ were not weaker than the reference display's, unlike the spokes of Display A. In any case, the presence of the spoke illusion in Display A but not in Display B suggests that spokes can be generated by retinal motion alone but not by perceived motion without retinal motion. The spokes are apparently generated at a stage where there is little distinction between motion across the retina caused by smooth pursuit and motion across the retina caused by stimulus motion in the world. We discuss possible implications of this finding for the neural basis of the illusion in the General Discussion.

\section{EXPERIMENT 2}

The results of Experiment 1 suggest that spokes are generated by a process early in the visual processing stream, before retinal motion due to eye movements is discounted. To functionally localize the process that generates the illusion even further, we went on to test whether this process utilizes chromatic contrast, as it does luminance contrast. If it does, then displays with chromatically defined figures should yield spokes different in color from the background that remain strong as long as the color contrast of the figure is high, regardless of the luminance contrast. To determine whether this was the case, in Experiment 2 a display with high chromatic contrast was used, and the effect of varying the display's luminance contrast was measured.

\section{Method}

Participants. Three observers with normal or corrected-to-normal vision, including two of the authors, participated. The two authors had extensive experience with these displays, and the other observer had extensive experience in psychophysical experiments.

Apparatus, Stimuli, Design, and Procedure. The stimuli were displayed on a calibrated 13-in. Apple color monitor and viewed from $\approx 75 \mathrm{~cm}$ away. Each observer's subjective equiluminance point was measured with both a minimally distinct border method and a minimum-flicker technique (Boynton, 1979). In the minimally distinct border method, the complex illusory contour figure of Parks (1980) was presented in saturated green (CIE coordinates: $x \approx 0.30$, $y \approx 0.58$ ) against a saturated red background (luminance $27 \mathrm{~cd} / \mathrm{m}^{2}$, CIE: $x \approx 0.61, y \approx 0.34$ ) at an eccentricity of $3.7^{\circ}$. In 10 separate trials, observers adjusted the luminance of the figure until the contours appeared minimally distinct. Additional estimates of the subjective equiluminance point were then made using a minimum-flicker method. Six disks, identical in size, hue, and eccentricity to the test disks of the experiment proper, were flickered at a rate of approximately $33 \mathrm{~Hz}$. The disks were initially presented at a random luminance, and observers adjusted the luminance to the point of minimal subjective flicker. Ten such trials were completed and the average of the 20 values yielded by the two methods was used as the subjective equiluminance point in the experiment.

In the main experiment, the observer viewed two disks situated on opposite sides of a fixation point. The disks, a "reference" disk and a "test" disk, subtended $\approx 2.9^{\circ}$, and their centers were $\approx 3.7^{\circ}$ from the fixation point. The background of the display was a saturated red (luminance $27 \mathrm{~cd} / \mathrm{m}^{2}$, CIE: $x \approx 0.61, y \approx 0.34$ ). The test disk was a saturated green (CIE: $x \approx 0.30, y \approx 0.58$ ) and was presented at a variety of luminances relative to the observer's subjective equiluminance point. The reference disk was white and was always presented at a luminance of $\approx 89 \mathrm{~cd} / \mathrm{m}^{2}(\mathrm{CIE}: x \approx .342, y \approx 0.339)$. The two disks revolved around the fixation point at a speed of $=8.6^{\circ} \mathrm{vi}$ sual angle $/ \mathrm{sec}\left(133^{\circ}\right.$ of the disk's orbit per second).

On each trial the test disk was presented at one of seven luminances, which spanned a range from darker than the background to brighter than the background. Relative to each observer's equiluminance point, these luminances were approximately $-21,-14,-7$, $0,7,14$, and $21 \mathrm{~cd} / \mathrm{m}^{2}$. On a given trial, the observer rated the strength of the spoke percept emanating from the test disk relative to the strength of the spoke from the reference disk on a scale of 0 to 9 . A rating of 9 meant that the test disk's spoke was as strong as the reference disk's spoke, and 0 meant that no spoke was perceived between the test disk and fixation. Thirty trials were conducted at each luminance offset, in pseudorandom order. After each trial, the entire display flickered rapidly between black and white to eliminate any visual persistence of the previous trial's stimulus and spoke.

\section{Results and Discussion}

According to our method, the luminance required for the green disk to appear subjectively equiluminant with the $27-\mathrm{cd} / \mathrm{m}^{2}$ background was: $29.3 \mathrm{~cd} / \mathrm{m}^{2}$ for A.S., $28.8 \mathrm{~cd} / \mathrm{m}^{2}$ for A.H., and $27 \mathrm{~cd} / \mathrm{m}^{2}$ for P.T. The $0-9$ rating scale was rescaled to $0-1$, and the mean and standard error of the ratings of relative spoke strength at each test disk luminance are plotted in Figure 3.

Observers reported that the illusion darkened or brightened the red background, but did not change its hue. The observers' ratings indicate that, for the luminance values sampled, spoke strength declined monotonically with decreasing luminance contrast. The response curves were fit with a model that assumed that spoke strength is a linear function of contrast, with luminance decrements $25 \%$ stronger than luminance increments (see, e.g., Sperling $\&$ Liu, 1999). The curve fits suggest that the luminances for minimum wake strength are within $0.13 \mathrm{~cd} / \mathrm{m}^{2}$ of each observer's subjective equiluminance point, which is within the error of the subjective equiluminance point estimates. Although the strength ratings indicate that the spoke's strength never declined to 0 , these nonzero ratings at equiluminance may have reflected color bleeding or simple color contrast that may have surrounded the equiluminant disk, both of which are confusable with a very weak spoke. In any case, the illusion's monotonic decline with decreasing luminance contrast and extreme 


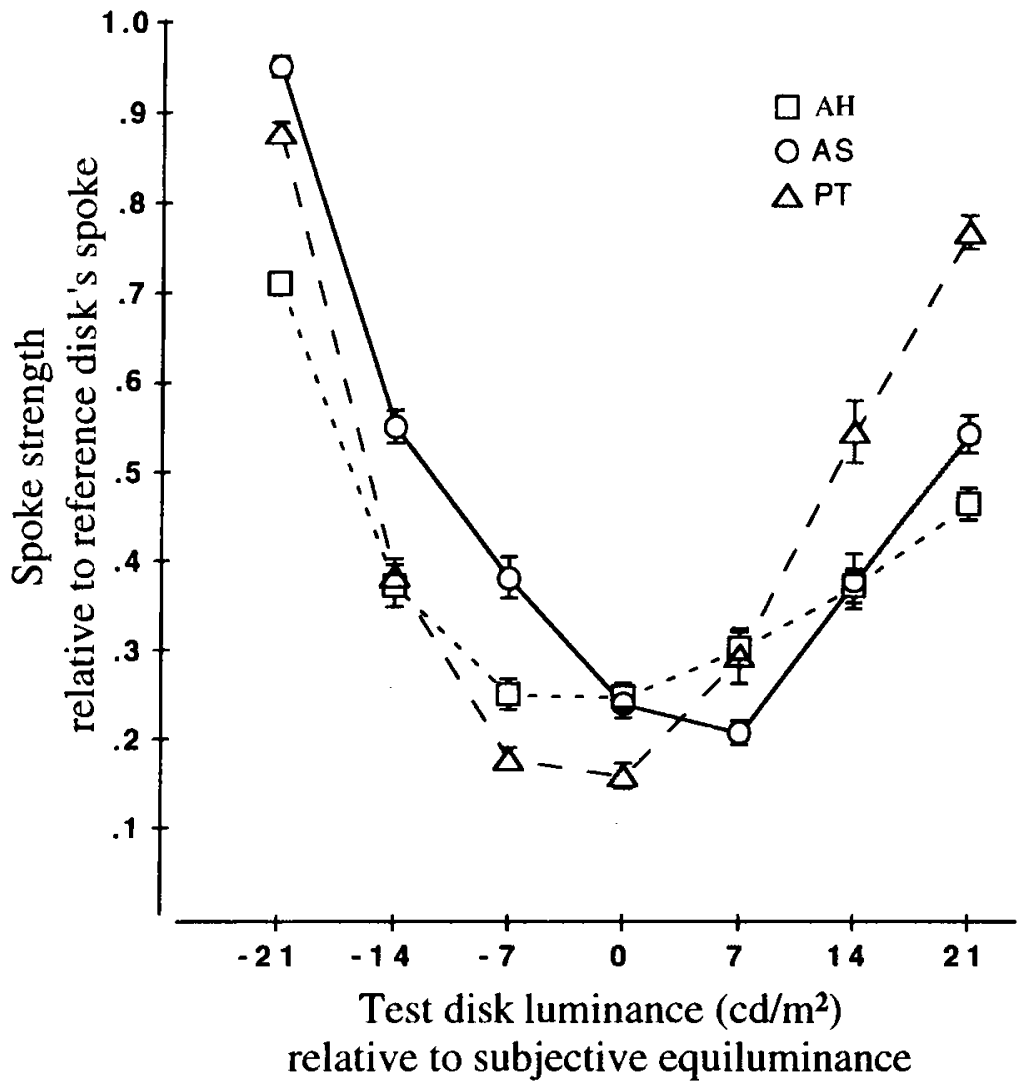

Figure 3. The results of Experiment 2. A saturated green "test" disk was presented on a red background and moved in a circular pattern around a fixation point. Opposite the green disk, a high-contrast white disk also orbited the fixation point. The green test disk was presented at a variety of luminances relative to each observer's subjective equiluminance point (established in a separate experiment). At each luminance offset, the observers rated the strength of the spoke induced by the green disk relative to the spoke induced by the white disk. Mean ratings, with the rating scale transformed to 0 to 1 scale, and standard errors are plotted at each luminance offset for each observer. The data are plotted so that each observer's subjective equiluminance point is at 0 .

weakness near equiluminance (despite high chromatic contrast) supports the notion that the spoke illusion originates at a stage where luminance, not color, dominates.

Further support for this conclusion is provided by additional informal investigations in which we varied color contrast widely but were unable to perceive an effect on the spokes' hue.

These results dissociate the spoke illusion from other, seemingly similar, illusions. Spokes seem similar to induced gratings because in both illusions, dark figures induce adjacent illusory bright areas and light regions induce adjacent illusory dark regions (McCourt, 1982). Simple brightness contrast also shares this property, as dark surrounds make a light figure appear even lighter and vice versa for light surrounds and dark figures. But unlike the spoke illusion, induced gratings and simple brightness contrast work well with figures defined by color alone (Kingdom \& Moulden, 1989; Krauskopf, Zaidi, \& Mandler, 1986; Zaidi, 1989). In addition, induced gratings and simple contrast displays are effective with stationary stimuli (Chevruel, 1848; McCourt, 1982), whereas spokes are visible only when the stimulus creates retinal motion. In comparison to these other illusions, the spokes seem to be caused by a more restricted process, one that occurs only with retinally moving, luminancedefined stimuli.

\section{GENERAL DISCUSSION}

Experiment 1 showed that spokes originate at a stage where motion across the retina due to smooth pursuit eye movements is not discounted. Experiment 2 showed that 
even in displays with high chromatic contrast, the strength of spokes declines with decreasing luminance contrast and becomes very weak or absent at subjective equiluminance.

Experiment 1 results suggest that the illusion is generated before the stage where motion due to eye movements is discounted. But exactly where in the brain is that? Neurophysiological researchers have attempted to determine in which brain areas neurons respond more to motion caused by external object movement than to motion caused by eye movements. Galletti and his collaborators found that $10 \%$ of the $\mathrm{Vl}$ cells in their sample responded more to externally caused movements (Galletti, Squatrito, Battaglini, \& Maioli, 1984), whereas $13 \%$ of V2 cells (Galetti, Battaglini, \& Aicardi, 1988) and 40\% of V3a cells (Galetti, Battaglini, \& Fattori, 1990) had this property (see Battaglini, Galetti, \& Fattori, 1996, for a review). This suggests that motion due to eye movements is discounted by some cells even at the earliest stage of cortical processing. However, the results reported by Erickson and Thier (1991; Thier \& Erickson, 1992) were quite different. In their experiments, all the directionselective cells that they investigated in V1, V2, and V3 responded equally well to motion across the retina caused by smooth pursuit and motion across the retina caused by external object movement. In addition, almost all cells in V4 and MT had this property, and it was only in dorsal MST that most cells responded preferentially to externally induced motion. Erickson and Thier criticized the work of Galletti et al. on methodological grounds, which led them to reject Galletti et al.'s interpretation of their results.

After further work determines more definitively how the brain discounts motion due to eye movements, specific neurophysiological implications of our experiments should follow. For the moment, it is at least clear that the proportion of cells that prefer externally caused motion increases as one ascends the visual processing stream, implying that early in cortical processing, motion processing is predominantly retina based. The existence of this predominantly retina-based motion processing stage has also been demonstrated with a motion aftereffect experiment (Anstis \& Gregory, 1965). In this experiment, the direction of the motion aftereffect was determined by the direction of the adapting retinal motion, even if the adapting motion was perceived to be in the opposite direction. Thus our results suggest that spokes originate early in the visual processing stream. Indeed, we cannot rule out the possibility that spokes are generated as early as the retina.

The finding that the strength of the spoke illusion is determined by luminance contrast does not localize the illusion's origin exactly, but it does further dissociate spokes from other illusions. Unlike the spoke illusion, grating induction and simple contrast displays are quite effective in the purely chromatic domain, and with stationary stimuli. As such, they may be by-products of processes that enhance spatial segmentation and provide gain control so that we can see more efficiently in dif- ferent environments. However, our findings that isoluminant displays do not result in a significant spoke illusion, that spokes only occur with moving displays, and that spokes are biased toward central fixation suggest that spokes reflect a more specific function. We hope that, by building on our results, subsequent work will uncover the role of the spoke-generating process in normal vision.

\section{REFERENCES}

Anstis, S. M., \& Gregory, R. L. (1965). The aftereffect of seen motion: The role of retinal stimulation and of eye movements. Quarterly Journal of Experimental Psychology, 17, 173-174.

Battaglini, P. P., Galletti, C., \& FatToRI, P. (1996). Cortical mechanisms for visual perception of object motion and position in space. Behavioural Brain Research, 76, 143-154.

BoYNTON, R. M. (1979). Human color vision. New York: Holt, Rinehart $\&$ Winston.

Bridgeman, B. (1995). A review of the role of efference copy in sensory and oculomotor control systems. Annals of Biomedical Engineering, 23, 409-422.

Chevruel, M. E. (1848). The principles of harmony and contrast of colors. New York: Van Nostrand \& Reinhold.

Erickson, R., \& Thier, P. (1991). A neuronal correlate of spatial stability during periods of self-induced visual motion. Experimental Brain Research, 86, 608-616.

Galletti, C., BattaglinI, P. P., \& AiCardi, G. (1988). "Real motion" cells in area V2 of behaving macaque visual cortex. Experimental Brain Research, 82, 67-76.

Galletti, C., Battaglini, P. P., \& Fattori, P. (1990). "Real-motion" cells in area V3A of macaque visual cortex. Experimental Brain Research, 82, 67-76.

Galletti, C., Squatrito, S., Battaglini, P. P., \& Maioli, M. G. (1984). "Real motion" cells in the primary visual cortex of macaque monkeys. Brain Research, 301, 95-110.

Holcombe, A. O., Macknik, S., Intriligator, J., Seiffert, A. E., \& TSE, P. U. (1999). Wakes and spokes: New motion-induced brightness illusions. Perception, 28, 1231-1242.

KINGDOM, F., \& MouldeN, B. (1989). Corner effect in induced hue Evidence for chromatic band-pass filters. Spatial Vision, 4, 253-266.

Krauskopf, J., ZAIDI, Q., \& MANDLER, M. B. (1986). Mechanisms of simultaneous color induction. Journal of the Optical Society of America, 3, 1752-1757.

MCCourT, M. E. (1982). A spatial frequency dependent grating-induction effect. Vision Research, 22, 119-134.

Parks, T. E. (1980). Letter to the editor. Perception, 9, 723.

SPERLING, G., \& LiU, Z-L. (1999). Unequal representation of black and white in human vision. Paper presented at the annual meeting of the Association for Research in Vision and Opthalmology, Ft. Lauderdale, Florida.

Thier, P., \& ERickson, R. (1992). Vestibular input to visual-tracking neurons in area MST of awake rhesus monkeys. In B. Cohen, D. L. Tomko, \& F. Guedry (Eds.), Sensing and controlling motion: Vestibular and sensorimotor function (Annals of the New York Academy of Sciences, Vol. 656, pp. 960-963). New York: New York Academy of Sciences.

TSE, P. (1997). Plasmas: A new class of motion-induced brightness illusions. Paper presented at the European Conference in Visual Perception.

WERTHEIM, A. H. (1987). Retinal and extraretinal information in movement perception: How to invert the Filehne illusion. Perception, 16, 299-308.

ZAIDI, Q. (1989). Local and distal factors in visual grating induction. $\mathrm{Vi}$ sion Research, 29, 691-697.

(Manuscript received December 8, 1998; revision accepted for publication February 11,2000 .) 